\title{
IMPLEMENTAÇÃO DE PROGRAMA DE ENFERMAGEM DE REABILITAÇÃO EM IDOSO COM FRAGILIDADE/SÍNDROME DE DESUSO - ESTUDO DE CASO
}

\section{IMPLEMENTACIÓN DE UN PROGRAMA DE ENFERMERÍA DE REHABILITACIÓN EN PERSONAS MAYORES CON FRAGILIDAD/SÍNDROME DE DESUSO - ESTUDIO DE CASO}

\author{
IMPLEMENTATION OF A REHABILITATION NURSING PROGRAM IN ELDERLY PEOPLE WITH \\ FRAGILITY/DISUSE SYNDROME - CASE STUDY
}

DOI 10.33194/rper.2020.v3.n2.5.5794 | Submetido 08.06.2020 | Aprovado 15/12/2020

\section{Ana Cristina Castanheira Gil ${ }^{1}$; ; Filipa Manuela Moreira Sousa ${ }^{1}$; ; Maria Manuela Martins ${ }^{2,3}$ (D) \\ 1 - Hospital CUF Porto; 2 - Escola Superior de Enfermagem do Porto; 3 - Grupo de Investigação NursID do CINTESIS}

\section{RESUMO}

Introdução: A pessoa com síndrome de desuso pode apresentar alterações do equilíbrio e uma condição de fragilidade, com consequente défice no desempenho das suas atividades de vida diária.

Objetivo: Identificar os ganhos sensíveis aos cuidados de enfermagem de reabilitação com um programa de atividades e exercícios terapêuticos na recuperação do equilíbrio e melhoria da fragilidade.

Método: Estudo de abordagem qualitativa, tipo estudo de caso. É apresentado o caso de uma pessoa idosa com síndrome de desuso admitida num internamento de adultos. Os princípios éticos em investigação foram cumpridos.

Resultados: Foram evidenciados ganhos no equilíbrio postural e na fragilidade após implementação de 9 sessões de exercícios terapêuticos e treino de equilíbrio.

Conclusão: A implementação de cuidados de enfermagem de reabilitação revelou-se eficaz na recuperação do equilíbrio corporal na pessoa com síndrome de desuso, assim como numa melhoria da fragilidade, otimizando a sua autonomia.

Palavras-chave: enfermagem de reabilitação, equilíbrio postural, exercícios terapêuticos, fragilidade, síndrome de desuso

\section{RESUMEN}

Introducción: La persona con síndrome de desuso puede presentar cambios de equilibrio y una condición de fragilidad, con el consecuente déficit en el desempeño de sus actividades de la vida diaria.

Objetivo: Identificar las ganancias sensibles al cuidado de enfermería rehabilitadora con un programa de actividades terapéuticas y ejercicios para recuperar el equilibrio y mejorar la fragilidad.

Método: Estudio cualitativo, estudio de caso. Se presenta el caso de un anciano con síndrome de desuso ingresado en un hospital de adultos. Se han seguido los principios éticos de la investigación.

Resultados: Se evidenciaron ganancias en el equilibrio postural y la fragilidad luego de la implementación de 9 sesiones de ejercicios terapéuticos y entrenamiento del equilibrio.

Conclusión: La implementación de los cuidados de enfermería rehabilitadora demostró ser efectiva en la recuperación del equilibrio corporal en personas con síndrome de desuso, así como en la mejora de la fragilidad, optimizando su autonomía.

Palabras clave: enfermería de rehabilitación, equilibrio postural, ejercicios terapéuticos, fragilidad, síndrome de desuso

\section{ABSTRACT}

Introduction: The person with disuse syndrome may present balance changes and a condition of fragility, with a consequent deficit in the performance of their activities of daily living.

Objective: To identify the gains that are sensitive to rehabilitation nursing care with a program of therapeutic activities and exercises to restore balance and improve frailty.

Method: Qualitative study, case study. The case of an elderly person with disuse syndrome admitted to an adult hospital is presented. The ethical principles in research have been followed. Results: Gains in postural balance and fragility were evidenced after the implementation of 9 sessions of therapeutic exercises and balance training. 
Conclusion: The implementation of rehabilitation nursing care proved to be effective in recovering body balance in people with disuse syndrome, as well as in improving frailty, optimizing their autonomy.

Keyword: rehabilitation nursing, postural balance, therapeutic exercises, fragility, disuse syndrome

\section{INTRODUÇÃO}

Cada vez mais se debate o tema do envelhecimento da população. O Instituto de Nacional de Estatística (INE) afirma inclusive que se prevê que em 2080, em cada 100 jovens, 317 serão idosos. 0 índice de envelhecimento em Portugal é de 157,4\%, com 153 idosos por cada 100 jovens, comparando-o ao índice de envelhecimento de 1965 , de $27,5 \%$ (1). Tendo em consideração este enquadramento populacional, assiste-se a uma preocupação social, económica e de saúde, de forma a encontrar respostas para esta nova realidade.

0 envelhecimento humano pode ser definido como o processo de mudança progressivo da estrutura biológica, psicológica e social dos indivíduos que, iniciando-se mesmo antes do nascimento, se desenvolve ao longo da vida ${ }^{(2)}$. Este não deve ser encarado como um obstáculo, mas sim como parte integrante do ciclo de vida, sendo que o desejável é que o idoso viva de forma saudável e autónoma, o máximo de tempo possível. Para que tal aconteça, é necessária uma reestruturação/adequação da atitude da população em geral, dos serviços de saúde, da aposta na formação de profissionais de saúde e dos apoios sociais, de forma a prevenir as fragilidades que frequentemente acompanham a pessoa idosa ${ }^{(2)}$.

São vários os conceitos para avaliar o envelhecimento, sendo a fragilidade um dos conceitos fundamentais. Está diretamente relacionada com o envelhecimento, e caracteriza-se pela diminuição das reservas fisiológicas e o aumento do declínio funcional, associado a múltiplas mudanças físicas ${ }^{(3)}$. A diminuição da massa e da força muscular, a exaustão, a alteração da marcha e do equilíbrio, a anorexia e a perda de peso progressiva (4), são algumas das mudanças físicas associadas ao envelhecimento e à síndrome da fragilidade no idoso. Embora a fragilidade esteja relacionada com a idade, não resulta exclusivamente do processo de envelhecimento, associa-se também à presença de comorbilidades, que se vão acumulando a este processo (4).

De acordo com vários estudos, os fatores associados à fragilidade são a idade, em que 3.9\% está compreendido entre 65-74 anos e $25 \%$ em idades de 85 anos ou mais, o sexo feminino, raça negra e baixo nível de escolaridade. Existe ainda um estudo que afirma que o uso de medicação excessiva, o elevado número de patologias e a perceção de saúde reduzida integram os fatores associados à fragilidade ${ }^{(4)}$.

A identificação precoce da fragilidade é fundamental, de forma a promover uma atuação mais antecipada e à utilização de intervenções mais eficazes. Para essa identificação é necessário a aplicação de instrumentos validados e confiáveis. Um grupo de pesquisadores do Canadá estudou uma forma de deteção da fragilidade no idoso e propôs uma escala para a sua avaliação: a escala de Edmonton Frail Scale (EFS), com nove domínios e onze itens. De acordo com o resultado, o grau de fragilidade varia de não frágil a fragilidade grave ${ }^{(6)}$.

Num estudo longitudinal realizado no Brasil com 262 participantes, 50,4\% eram frágeis e 21,0\% vulneráveis (5). De forma a combater estes números é primordial a aposta na reabilitação do idoso. A prática de exercício físico nos membros inferiores é considerada como o mais eficaz método para preservar a mobilidade, prevenindo desta forma o declínio funcional do idoso ${ }^{(4)}$.

A mobilidade é a capacidade de se locomover, é uma função complicada e composta de múltiplas ações, que dependem de uma integração de múltiplas características: físicas, cognitivas e psicológicas ${ }^{(7)}$. Em 1986 Tinetti desenvolveu uma escala para avaliar o equilíbrio estático e dinâmico e a mobilidade, que se caracteriza pela não necessidade de equipamentos sofisticados, sendo confiável para detetar mudanças significativas durante a marcha. A utilização deste instrumento de avaliação tem importantes implicações na qualidade de vida da pessoa com fragilidade, uma vez que possibilita ações preventivas, assistenciais e de reabilitação.

A evidência de que a estabilidade postural diminui com a idade, está descrita na bibliografia. A atividade física, com exercício aeróbico e de força, pode prevenir o declínio de muitas estruturas relacionadas com a manutenção do equilíbrio. A força muscular, a capilarização celular, a inervação das fibras musculares ativas e a flexibilidade em idosos, pode ser trabalhada e melhorada através de um programa de exercícios de força ${ }^{(8)}$. Assim, torna-se fundamental a implementação de um programa de exercícios de forma a promover o equilíbrio.

\section{MATERIAL E MÉTODOS}

O estudo de caso, como método de pesquisa, permite ao investigador estudar fenómenos individuais ou de grupo, em contexto real, com o objetivo de explorar, descrever e explicar um evento com base no problema de investigação de forma a compreender claramente o fenómeno, recorrendo a várias fontes de evidência ${ }^{(9)}$. Indo de encontro ao citado, recorreu-se aos referenciais de Yin e Stake para a elaboração do presente estudo de caso.

O estudo descreve o caso de uma idosa com alteração do equilíbrio postural e síndrome de fragilidade devido a internamento prolongado em unidade de cuidados intensivos, por pneumonia e demonstra um programa de reeducação funcional do equilíbrio desenvolvido em domicílio, evidenciando os resultados obtidos, com diminuição da fragilidade. A colheita de dados foi realizada através da anamnese e consulta do processo clínico. 
O programa de reabilitação, decorreu durante o mês de março de 2019, desde a admissão no serviço de internamento até ao domicílio, durante 9 dias. Foram monitorizados os resultados alcançados durante as sessões e para o presente estudo estão representados na tabela 3 e 4 . A pessoa envolvida foi designada por "Sr. ${ }^{a}$ M.", salvaguardando a confidencialidade dos dados e anonimato da mesma. A Sr. ${ }^{a} \mathrm{M}$ foi informada dos direitos na participação do estudo e foi obtido o consentimento livre e esclarecido antes da colheita de dados.

De forma a avaliar, quantificar e evidenciar o equilíbrio e a síndrome da fragilidade recorreu-se a instrumentos de medida, nomeadamente à Escala de Tinetti por ser de fácil aplicabilidade e não requerer equipamentos sofisticados e à Escala da fragilidade de Edmonton adaptada.

\section{APRESENTAÇÃO DO CASO}

\section{Anamnese}

O caso em estudo corresponde a uma pessoa de 65 anos de idade, género feminino, caucasiana, de nacionalidade portuguesa. É casada, tem 3 filhos adultos, financeiramente autónomos e 3 netos. É médica reformada.

Admitida a 6 de fevereiro de 2020, por insuficiência respiratória com hipoxemia grave, fumadora de 20 cigarros por dia desde os 15 anos. Sem alergias medicamentosas conhecidas. Recorre ao atendimento permanente com quadro de tosse seca com início há 5 dias, mialgias, astenia e dispneia para pequenos esforços, já tendo tomado azitromicina $500 \mathrm{mg}$ durante três dias.: ph7,46; pO2 40,9; pCO2 34,8 e HCO3 24,1. Realiza Angio TAC sem evidência de trombo embolismo pulmonar (TEP), mas com vidro despolido e infiltrados bilaterais a sugerir síndrome de dificuldade respiratória aguda (ARDS). Colheu hemoculturas e Influenza A. Normotensa e normocárdica, apirética e com SPO2 de $84 \%$, realizada gasimetria.

Internada na unidade de cuidados intensivos (UCIP), ajustado $\mathrm{FiO2}$, realizada pesquisa de antigenúrias, dando início a antibiótico. Realizou eco transtorácico e fez colheita de sangue para Peptideo Natriurético Cerebral (BNP.)

Relativamente à evolução nos 20 dias de internamento na UCIP, nos primeiros 13 dias esteve com entubação orotraqueal, sob ventilação mecânica (estratégiaprotective lung ventilation + prone position). Extubada a 19 de fevereiro sem intercorrências. Cumpriu 9 dias de ceftriaxone $2 \mathrm{gr}$ por dia mais 10 dias de oseltamivir $75 \mathrm{mg} 2 x /$ dia, por pneumonia, associada à ventilação. Cumpriu 6 dias de Piperacillin/Tazobactam 4g/0.5g e 2 dias de gentamicina. Posteriomente com isolamento de Pseudomonas aeruginosa resistente ao Piperacillin/Tazobactam 4g/0.5g e cefftazidima e sensível a Meropenem e levofloxacina, fez 7 dias de levofloxacia.

Miopatia doente crítico sob fisioterapia, desde 19 de fevereiro, dia de extubação orotraqueal. No Início pouco colaborante e com períodos de desorientação no tempo e espaço, realizada mobilização passiva dos quatro membros. Não cumpria ordens simples. A partir de 24 de fevereiro mais colaborante com mobilização ativa dos quatro membros e realiza levante, sem equilíbrio estático e dinâmico. No dia 27 de fevereiro foi transferida para o internamento de adultos em cadeira de rodas. Iniciou treino de marcha com andarilho e no dia 28 teve alta para domicílio, após iniciar as primeiras avaliações e o primeiro treino do programa de reabilitação. Por consequência, estes fatores levaram à perda de equilíbrio e capacidade de marcha. Neste processo, a Sr. ${ }^{a} \mathrm{M}$. perdeu a autoestima e capacidade de realizar as suas atividades de vida diária, ficando numa situação de fragilidade associada à cognição alterada, sentimento de tristeza e performance funcional.

Apresenta como antecedentes médicos: depressão, psoríase, obesidade, atopia cutânea e síndrome vertiginoso e como antecedentes cirúrgicos: colecistectomia em 2017, herniorrafia discal (L2) e prótese total da anca à direita. Medicada com: fluoxetina $20 \mathrm{mg}$, aldactone $25 \mathrm{mg}$, olcadil $2 \mathrm{mg}$, metformina $1000 \mathrm{mg}$ e livazo $2 \mathrm{mg}$.

Semidependente nas AVD no momento da alta para domicílio. Com suporte familiar e empregada doméstica que ajudam na satisfação das necessidades e incentivam à adesão ao programa de reabilitação e consequente recuperação.

\section{Avaliação de Enfermagem de Reabilitação}

Para a implementação de um programa de reabilitação é necessário incluir dados obtidos através da anamnese, da observação dos exames complementares de diagnóstico e da implementação de instrumentos de medida.

A avaliação e seguimento da Sr. ${ }^{a} \mathrm{M}$. iniciou-se no internamento hospitalar, no momento da alta, tendo continuidade e término no domicílio, com a duração de 9 dias, sendo que a denominação para os dias será: D1 até D9. Posteriormente foi necessário finalizar o processo de reabilitação devido à pandemia por COVID19.

O início do programa teve um primeiro momento, a realização de uma reunião familiar pré-alta, uma vez que era preciso reforçar à família o novo estado de saúde do seu familiar, de forma a perceber quem cuidaria em casa. E ainda para informar sobre os recursos existentes na comunidade, e por fim, para apresentar a proposta do programa de reabilitação, tudo para uma melhoria da continuidade de cuidados. Após aceitação e consentimento para participar no estudo delineamos o plano.

Com base na avaliação do equilíbrio e da fragilidade foi possível identificar diagnósticos de enfermagem e determinar os ganhos sensíveis aos cuidados de enfermagem de reabilitação. 


\section{Avaliação do equilíbrio}

o Índice de Tinetti classifica o equilíbrio sentado e em pé, a marcha, assim como a mudança de direção. Avalia o equilíbrio estático na cadeira, através da avaliação do equilíbrio sentado, levantar-se, equilibrar imediato, equilíbrio em pé com os pés paralelos, pequenos desequilíbrios na mesma posição, fechar os olhos na mesma posição, volta de $360^{\mathrm{a}}$, apoio unipodal e sentarse. Na avaliação do equilíbrio dinâmico - marcha, avalia-se o início da marcha, a largura e altura do passo do pé direito e esquerdo, simetria do passo, continuidade do passo, percurso de 3 metros, estabilidade do tronco e base de sustentação durante a marcha. A Sr. ${ }^{a}$ M. apresentou um score inicial de 19/28 aquando da implementação do programa de reabilitação, com alterações mais evidentes no equilíbrio estático na cadeira. Ao longo do programa houve ganhos no equilíbrio estático e dinâmico com score de $27 / 28$.

\section{Avaliação da fragilidade}

Para avaliação da fragilidade fez-se uso da escala da fragilidade de Edmonton adaptada, onde se avalia a cognição, o estado de saúde geral, a independência funcional, o suporte social, o uso de medicação, a nutrição, o humor, a continência e a performance funcional, sendo que na sua interpretação 0 pontos corresponde a não frágil, de 1-2 pontos pré-frágil e mais de 3 pontos, frágil. No início do programa a Sr. ${ }^{a} \mathrm{M}$. apresentava-se frágil e no $D 9$ já se encontrava num estado de pré-frágil.

\section{Diagnóstico de Enfermagem}

No âmbito do programa de reabilitação foi identificado o diagnóstico equilíbrio corporal comprometido relacionado com o foco "equilíbrio corporal" e desenvolvidas intervenções de enfermagem com base no padrão documental dos cuidados de Enfermagem da Especialidade de Enfermagem de Reabilitação ${ }^{(10)}$.

Tabela 1. Diagnósticos e Intervenções de enfermagem

Diagnóstico de Enfermagem

\begin{tabular}{|l|}
\hline Diagnóstico de Enfermagem \\
\hline Equilíbrio corporal comprometido \\
Potencial para melhorar conhecimentos \\
sobre técnica de treino de equilíbrio
\end{tabular}

Potencial para melhorar capacidade para usar técnica de equilíbrio corporal

\section{Intervenções de Enfermagem}

Aplicar estratégias de correção postural:

- colocar-se junto do lado oposto ao desequilíbrio, utilizar estímulos tácteis

toque com dois dedos no ombro homolateral ao desequilíbrio;

- técnica de reeducação postural ao espelho;

Estimular a manter equilíbrio corporal, através da correção postural;

Executar técnica de treino de equilíbrio deitado, sentado e em pé:

- exercício do gancho e ponte,

- dissociação de cinturas,

- rolar,

- balancear dentro dos eixos anatómicos e retomar à posição de origem,

- facilitação cruzada,

- coordenação de movimentos

Orientar na técnica de treino de equilíbrio

Ensinar sobre técnica de equilíbrio corporal sentado e em pé; Instruir sobre técnica de equilíbrio corporal sentado e em pé;

Treinar sobre técnica de equilíbrio corporal sentado e em pé
Para além das intervenções de enfermagem planeadas, foi definido um plano de treino para fortalecimento muscular e potencialização dos resultados.

Seguindo as recomendações mais recentes para exercícios a idosos e os objetivos delineados para a Sr. ${ }^{a}$ M., delineamos o seguinte programa de exercícios, ressaltando que o tipo de exercício deve focar-se em atividades funcionais específicas, tendo em conta as necessidades e os objetivos do doente ${ }^{(11)}$. O plano de intervenção foi elaborado tendo em conta o tipo de exercício, a intensidade, a duração e a frequência ${ }^{(12)}$.
O início de cada exercício foi exemplificado pelas investigadoras, prosseguindo a verificação da execução, corrigindo e reforçando positivamente o esforço e a execução do mesmo. As sessões foram intensificando conforme a capacidade da Sr. ${ }^{a} \mathrm{M}$. de forma a evitar o cansaço e desmotivação. Nos primeiros dois dias existiu a necessidade de mais reforço, atenção e diminuição do número de repetições de cada exercício. Com a evolução do programa a intensidade dos exercícios foi aumentando e consequentemente a Sr. ${ }^{a}$ M. aumentou a capacidade de resistência e perseverança em realizar corretamente os exercícios propostos. 
Tabela 2. Programa de Exercícios

Tempo

$10^{\prime}$

aquecimento

30

fortalecimento

e equilíbrio

10

\section{Exercício respiratórios}

Sentada contração isométrica dos glúteos, $3 \times 10$ repetições (rep.)

Sentada exercícios de dorsiflexão do tornozelo, $3 \times 10$ rep.

Apoiada nas costas da cadeira em pé pontas dos pés, $3 \times 10$ rep.

Apoiada nas costas da cadeira em calcanhares. $3 \times 10$ rep.

Apoiada nas costas (3x 10 rep.):

- abdução-adução coxofemoral

- flexão-extensão coxofemoral

- flexão-extensão joelho

Movimento de flexão e abdução/adução a 3 pontos, 3X10 rep. para cada perna

Exercícios levantar e sentar sem apoio, 3X 10 rep.

Exercícios de olhos fechados dedo nariz , 1x10 rep. para cada mão

Sentada flexão-extensão dos MS com halteres $0,5 \mathrm{Kg}, 3 \times 10$ rep. cada membro

Marcha controlada com andarilho , 4x, 3 metros

Marcha controlada , 4x, 3 metros

Marcha livre com andarilho, 4x, 3 metros

Marcha livre, 4x, 3 metros

Treino subir e descer escadas , 4x, 15 degraus

Exercício respiratórios

arrefecimento Alongamentos (membros superiores, membros inferiores, coluna)
Cadeira

Marcadores

Material

Cadeira

Tapete treino de equilíbrio dinâmico

Andarilho

Tapete treino de equilíbrio

Corredor

Corredor

Escadas

\section{RESULTADOS}

O programa de reabilitação foi instituído durante 9 dias durante o mês de março de 2020,5 sessões de 45 minutos na primeira semana e 4 sessões de 45 minutos na segunda semana. Por causa da pandemia foi necessário terminar o programa inicialmente planeado para 20 sessões mais cedo.

Está descrito numa revisão bibliográfica que o fortalecimento muscular melhora a força dos músculos, a mobilidade funcional e o equilíbrio de indivíduos idosos. Estes autores analisaram estudos que estabeleciam correlações entre programas de fortalecimento muscular e o desempenho funcional de idosos no equilíbrio e na marcha, tendo conseguido apurar que os idosos mais fragilizados obtiveram melhoras mais significativas na função ${ }^{(13)}$. No idoso frágil o uso de treino de força é recomendado e considerado de extrema importância pela American College of Sports Medicine ${ }^{(14)}$.

\section{Avaliação do equilíbrio estático e dinâmico}

A evolução do equilíbrio foi avaliada através da escala de Tinetti, avaliando diariamente o equilíbrio estático e dinâmico, partindo do pressuposto que no final do plano de reabilitação a $\mathrm{Sr}^{{ }^{a}} \mathrm{M}$. obtivesse ganhos, apresentado um score total da soma da avaliação do equilíbrio estático e dinâmico de 28 .

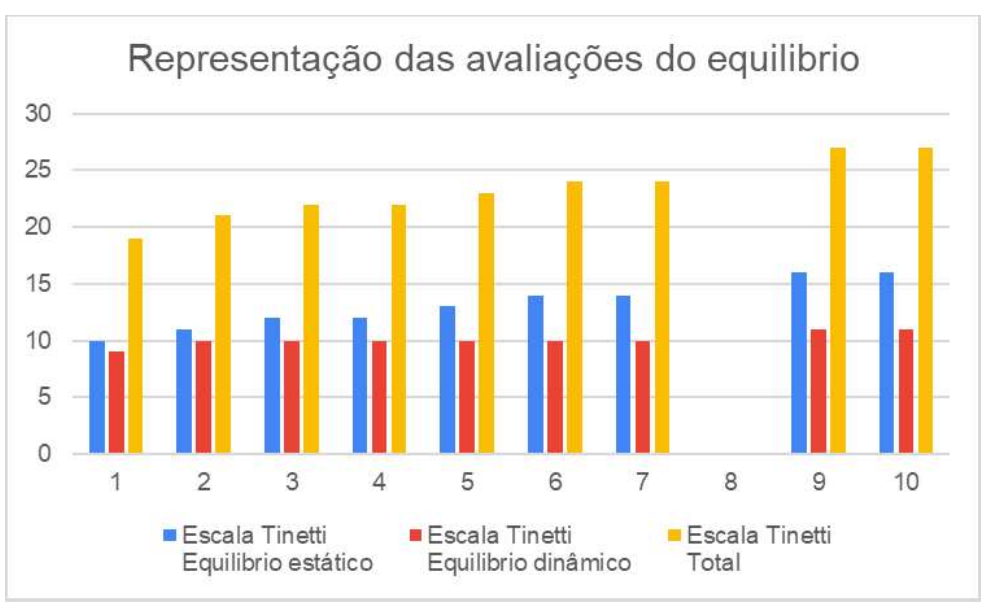

Gráfico 1. Avaliação do equilíbrio corporal através da escala de Tinetti

No D1 da aplicação da escala de Tinetti a doente apresentava um score de 10 no equilíbrio estático e um score de 9 no equilíbrio dinâmico, somando um total de 19. No D9 da aplicação da escala de Tinetti, a doente já apresentava um score de 16 no equilíbrio estático e um score de 11 no equilíbrio dinâmico, somando um total de 27.0 item em que não se obteve o score total da escala de Tinetti, foi relativo ao percurso de 3 metros previamente marcados, na medida em que a $\mathrm{Sr} .{ }^{\mathrm{a}}$ M.P ainda se desvia ligeiramente do percurso marcado. A média final da avaliação da escala de Tinetti foi de 23.

Consideramos que se a aplicação do programa de reabilitação se prolongasse por um período maior de 
tempo, este valor se aproximava mais do score pretendido ${ }^{(15)}$, tendo em consideração as avaliações a partir do D6. De realçar os seguintes aspetos:

- D1 até ao D4 a Sr.a M.P necessitava da ajuda do marido para o autocuidado higiene e que a partir do D5 já era capaz de desempenhar esta tarefa autonomamente;

- D1 até a D6 não era capaz de subir e descer as escadas da sua habitação. A partir de D7 já o fazia com recurso ao uso do corrimão;

- D8 já era capaz de se deslocar ao café com ajuda de terceiros, e no D9 já fez o mesmo percurso, sozinha, sem perda de equilíbrio.

Tabela 3. Score da avaliação do Índice de Tinetti

\begin{tabular}{c|c|c|c|c|c|c|c|c|c|} 
Dia & D1 & D2 & D3 & D4 & D5 & D6 & D7 & D8 & D9 \\
$\begin{array}{c}\text { Equilíbrio } \\
\text { Estático: } \\
\text { Cadeira }\end{array}$ & $10 / 16$ & $11 / 16$ & $12 / 16$ & $12 / 16$ & $13 / 16$ & $14 / 16$ & $14 / 16$ & $16 / 16$ & $16 / 16$ \\
$\begin{array}{c}\text { Equilíbrio } \\
\text { Dinâmico: } \\
\text { Marcha }\end{array}$ & $9 / 12$ & $10 / 12$ & $10 / 12$ & $10 / 12$ & $10 / 12$ & $10 / 12$ & $10 / 12$ & $11 / 12$ & $11 / 12$ \\
\hline Total & $\mathbf{1 9 / 2 8}$ & $\mathbf{2 1 / 2 8}$ & $22 / 28$ & $22 / 28$ & $23 / 28$ & $24 / 28$ & $24 / 28$ & $27 / 28$ & $27 / 28$ \\
\hline
\end{tabular}

\section{Avaliação da fragilidade}

Para avaliação da fragilidade, foi aplicada a escala de da fragilidade de Edmonton adaptada, no D1 e no D9, em que na interpretação da escala, 0 pontos corresponde a não frágil, de 1-2 pontos pré frágil e mais de 3 pontos frágil. Através da análise dos resultados obtidos foi possível perceber que a Sr. ${ }^{a} \mathrm{M}$. passou de um estado de fragilidade, com 9 pontos no D1, para um estado de pré fragilidade, com 2 pontos no D9. Embora os resultados sejam bastante positivos, com evidência da eficácia da implementação de programas de reabilitação no síndrome da fragilidade, consideramos que a Sra M.P. tem potencial para evoluir para um estado de não frágil, na medida em que os pontos que lhe foram atribuídos no D9, foram relativos à independência funcional (utilizar transportes), em que não houve possibilidade de avaliar efetivamente se a doente era capaz de o fazer de forma autónoma, sendo que a resposta da Sr. ${ }^{a} \mathrm{M}$. foi baseada no que ela considerava ser ou não capaz, e performance funcional, demorando ainda 11-20s no trajeto de 3 metros. Com aumento do período de implementação do programa de reabilitação, e tendo em consideração a avaliação no D1 e comparação deste parâmetro no D9, consideramos que seria então possível que a cliente passasse do estado de pré fragilidade para não frágil.

Escala da fragilidade inicio e fim

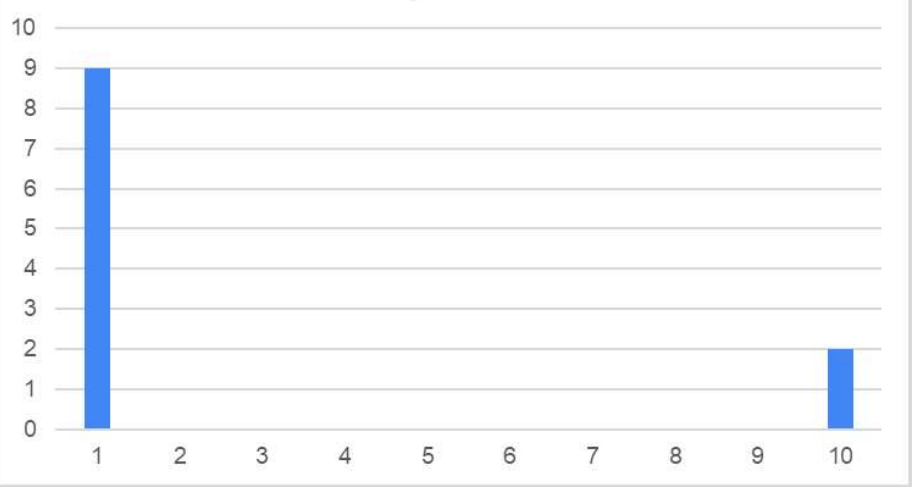

Gráfico 2. Avaliação da fragilidade através da escala de Edmonton adaptada

Tabela 4. Escala de fragilidade de Edmonton adaptada

\begin{tabular}{|c|c|c|c|c|c|c|}
\hline Domínio & Item & 0 pontos & 1 ponto & 2 pontos & D1 & D9 \\
\hline Cognição & $\begin{array}{l}\text { Imagine que este círculo é um relógio. Coloque os números } \\
\text { nas posições corretas e depois marque as horas e depois } \\
\text { marque as horas onze e dez }\end{array}$ & sem erros & $\begin{array}{c}\text { erros } \\
\text { menores no } \\
\text { espaçamento }\end{array}$ & $\begin{array}{l}\text { outros } \\
\text { erros }\end{array}$ & 1 & 0 \\
\hline \multirow{2}{*}{$\begin{array}{l}\text { Estado } \\
\text { geral de } \\
\text { saúde }\end{array}$} & No ano passado quantas vezes esteve internado no hospital? & 0 & $1-2$ & $\gg 2$ & 0 & 0 \\
\hline & No geral, como descreveria a sua saúde? & $\begin{array}{c}\text { excelente, } \\
\text { muito boa, boa }\end{array}$ & $\begin{array}{l}\text { mais ou } \\
\text { menos }\end{array}$ & fraca & 2 & 0 \\
\hline $\begin{array}{l}\text { Indepen- } \\
\text { dência } \\
\text { funcional }\end{array}$ & $\begin{array}{l}\text { Em quantas das seguintes atividades necessita de } \\
\text { ajuda?(preparar refeições, fazer compras, utilizar } \\
\text { transportes, usar o telefone, arrumar a casa, cuidar da roupa, } \\
\text { gerir o dinheiro, tomar medicação) }\end{array}$ & $0-1$ & $2-4$ & $5-8$ & 2 & 1 \\
\hline $\begin{array}{l}\text { Suporte } \\
\text { social }\end{array}$ & $\begin{array}{l}\text { Quando precisa de ajuda, há alguém com quem possa contar } \\
\text { para o ajudar? }\end{array}$ & sempre & Às vezes & Nunca & 0 & 0 \\
\hline \multirow{2}{*}{$\begin{array}{l}\text { Uso } \\
\text { medicação }\end{array}$} & Toma cinco ou mais medicamentos regularmente & Não & Sim & & 0 & 0 \\
\hline & Costuma esquecer-se de tomar a medicação? & Não & Sim & & 0 & 0 \\
\hline Nutrição & $\begin{array}{l}\text { Perdeu peso ultimamente que fizesse notar diferença na } \\
\text { roupa? }\end{array}$ & Não & Sim & & 1 & 0 \\
\hline Humor & Costuma sentir-se deprimido? & Não & Sim & & 1 & 0 \\
\hline Continência & Por vezes acontece-lhe perder urina quando não quer? & Não & Sim & & 0 & 0 \\
\hline $\begin{array}{l}\text { Perfor- } \\
\text { mance } \\
\text { funcional }\end{array}$ & $\begin{array}{l}\text { Sente-se nesta cadeira com os braços ao longo do corpo. } \\
\text { Quando eu disser "agora", levante-se e caminhe a um ritmo } \\
\text { normal até à marca que se encontra no chão (a cerca de } 3 \\
\text { metros), depois regresse para a cadeira e sente-se. }\end{array}$ & $0-10 \mathrm{~s}$ & $11-20 s$ & $\begin{array}{l}>20 \text { s ou } \\
\text { paciente } \\
\text { recusa } \\
\text { sequer } \\
\text { ajuda }\end{array}$ & 2 & 1 \\
\hline \multicolumn{2}{|l|}{ Total } & & & & 9 & 2 \\
\hline
\end{tabular}




\section{DISCUSSÃO}

Em Portugal, pelo número crescente de pessoas idosas, cada vez mais se assiste a altas hospitalares de pessoas com mais de 65 anos, que requerem continuidade de cuidados, apoio na reabilitação e reinserção social (16). No caso da Sr. ${ }^{a}$ M., o planeamento da implementação do programa de reabilitação com a doente, assim como a discriminação de todo o processo em domicílio foi fundamental para a sua total disponibilidade. Por ter empregada doméstica durante um grande período do dia, não foi difícil programar a alta para domicílio de acordo com o definido pela equipa médica. Com o planeamento de alta hospitalar pretende-se garantir a continuidade dos cuidados e a utilização eficiente dos recursos tanto hospitalares como da comunidade (16). Tem também como finalidade uma transferência segura, evitando reinternamentos, aumentos de custos para o sistema de saúde e ultrapassar as possíveis dificuldades do doente e família ${ }^{(17)}$.

A fragilidade é descrita como uma síndrome, em que se identifica perda de peso involuntária, exaustão, fraqueza, diminuição da velocidade da marcha e do equilíbrio e diminuição da atividade física, que pode levar a um risco de queda elevado, declínio funcional, hospitalização e morte ${ }^{(17)(18)(19)}$. No estudo de caso, a SR. ${ }^{a} M$. manifesta exaustão, fraqueza, diminuição da velocidade da marcha e do equilíbrio, o que leva a sua inclusão nesta síndrome. 0 idoso apresenta síndrome da fragilidade quando quatro dos seguintes critérios estão presentes: idade igual ou superior a 80 anos, depressão, instabilidade de equilíbrio ou marcha, diminuição da força de preensão palmar, uso de medicação, diminuição da força das articulações dos ombros e joelhos, défices dos membros inferiores e déficit visuais (19)(20). No caso da Sr. ${ }^{a}$ M. , apresenta como critérios para a síndrome da fragilidade: depressão, instabilidade no equilíbrio e marcha e uso de medicação. Tem maior prevalência no sexo feminino, o que vai de encontro ao género da nossa doente em estudo, e a sua característica essencial é a noção de risco decorrente da instabilidade (18)(19). A Sr. ${ }^{a}$ M. tem alta do internamento para domicílio com apoio de andarilho, por não se sentir segura para caminhar sem ele.

Para avaliar o grau de fragilidade utilizou-se a escala de Edmonton Frail Scale (EFS). De acordo com o valor obtido, o grau de fragilidade varia de fragilidade grave, pré-frágil e não frágil (6)(18). A Sr. ${ }^{a} \mathrm{M}$. passou de um estado de fragilidade para um estado de préfragilidade, pela interpretação dos resultados obtidos da aplicação da escala. Esta melhoria deveu-se à aplicação de um programa de reabilitação. Programas de reabilitação adequados ajudam na prevenção e até mesmo na reversão de quadros de fragilidade. Existe também evidência da eficácia dos programas de reabilitação na melhoria nos domínios do equilíbrio, marcha e força dos membros inferiores e superiores (18)(20)(12). Através da interpretação dos resultados obtidos, foi possível confirmar ganhos em saúde, com aumento do equilíbrio e melhoria do estado de fragilidade, fruto da aplicação de um programa de reabilitação, o que vai ao encontro aos resultados obtidos em diferentes estudos ${ }^{(17)(18)}$.
Pela análise dos estudos encontrados, os programas de reabilitação implementados têm duração em média de 10 a 12 semanas, com frequência de $3 x$ semana, com duração média de 60 minutos e com o número de repetições de 3 séries de 10 . Os programas de reabilitação instituídos são semelhantes entre si, incidindo no aquecimento, fortalecimento muscular, treino do equilíbrio, alongamento e relaxamento (15)(21)(17)(18)(20)(22). Recomendam a inspiração antes do levante e a expiração durante a carga ${ }^{(18)}$. A aplicação do programa de reabilitação foi inicialmente planeada para um período de 20 dias, de segunda a sexta feira, durante um mês, tendo o seu início no internamento, com continuidade no domicílio. A literatura encontrada refere que a frequência deve ser de $3 x$ semana, com um período de repouso de 48 horas entre cada sessão, para recuperação muscular ${ }^{(18)}$, ao contrário do que foi instituído no nosso programa de reabilitação. Este facto não comprometeu a adesão da Sr. ${ }^{a}$ M., mas realça-se o facto de haver da sua parte informação de "dor muscular" quando iniciava o programa de reabilitação. Devido à pandemia COVID-19, foi necessário reduzir a sua implementação para 9 dias. Mesmo com esta redução para metade do tempo planeado e muito longe da média descrita nos vários estudos encontrados, os resultados obtidos foram positivos, com ganhos em saúde evidentes.

O equilíbrio foi avaliado através da aplicação da escala de Tinetti, avaliando diariamente o equilíbrio estático e dinâmico. Estudos referem que os exercícios para treino de equilíbrio podem ser estáticos e/ou dinâmicos que envolvam alterações no input visual, mudanças na superfície de sustentação e redução na base de sustentação, com aumento gradual do nível de dificuldade e complexidade das posições (18)(15)(21). Para o estudo de caso foi usado o tapete de marcha, para avaliar o equilíbrio, onde se aplicaram os seguintes exercícios: rodar 3600 , caminhar com os pés juntos, caminhar de costas, caminhar de lado, caminhar em bicos de pés e caminhar com os calcanhares. Utilizouse também um exercício de 4 pontos que consistia na seguinte sequência: colocação de 5 discos no chão, um central, um à frente da doente, outro atrás e 2 nas laterais, um à direita e outro à esquerda. Na realização do exercício a doente executava exercícios de flexão até aos pontos marcados e extensão até ao disco central, sem apoio, passando por todos os discos.

Através da interpretação dos resultados obtidos, foi possivel perceber uma melhoria significativa no equilíbrio, passando de um total de 19 pontos (num total de 28) da avaliação do D1, para um total de 27 pontos (num total de 28 ) da avaliação do D9, indo de encontro à bibliografia encontrada, onde há evidência de melhoria significativa na força muscular, equilíbrio, coordenação e cadência (17)(15)(20).

Com a evolução dos dias, denota-se uma antecipação da realização dos exercícios, por conhecimento do plano de reabilitação, e melhoria significativa do equilíbrio, tendo desta forma maior facilidade na execução e concretização dos exercícios. 
A Sr. ${ }^{a} \mathrm{M}$. percebeu a importância da sua dedicação para atingir os seus objetivos pessoais, sendo que um deles seria ir ao Algarve após conclusão do programa. A sua não concretização não se deveu a falha no programa, mas à situação de pandemia atual do país. É importante salientar também que, após o primeiro dia de aplicação do programa de reabilitação no domicílio foi necessário reajustá-lo, tendo em consideração as barreiras arquitetónicas da habitação e a capacidade da $\mathrm{Sr}^{\mathrm{a}}{ }^{\mathrm{M}} \mathrm{M}$. para executar todos os exercícios com sucesso.

\section{CONSIDERAÇÕES FINAIS}

Com este estudo foi possível mobilizar e aprofundar conhecimentos acerca do papel do enfermeiro de reabilitação na capacitação da pessoa com síndrome de desuso e fragilidade. Através de um programa de exercícios que promovem o fortalecimento muscular foi possível promover a autonomia da $\mathrm{Sr} .{ }^{a} \mathrm{M}$ e a restituição da sua independência para as AVD e atividades de lazer.

A limitação encontrada para a realização deste estudo de caso relaciona-se com a pouca experiência das investigadoras na realização e concretização de programas de reabilitação, assim como na capacidade de análise de todos os focos inerentes à pessoa com síndrome de desuso e fragilidade.

Sugere-se em estudos posteriores que se implementem programas de reabilitação num período de tempo mais alargado e com incidência também noutros focos de enfermagem como a avaliação da força muscular e o andar, de forma a termos uma avaliação mais completa da doente.

\section{REFERÊNCIAS BIBLIOGRÁFICAS}

1. Indicadores de envelhecimento-526 [Internet]. Pordata: FFMS; 2018 [cited 2020 Apr 16]. Available from: https://www. pordata.pt/Portugal/Indicadores+de+envelhecime nto-526

2. Programa nacional para a saúde das pessoas idosas: Divisão de Doenças Genéticas, Crónicas e Geriátricas [Internet]. DGS; 2006 [cited 2020 Apr 16]. Available from: https: / /www.dgs.pt/documentos-e-publicacoes/programanacional-para-a-saude-das-pessoas-idosas-pdf.aspx

3. Fhon Jack Roberto Silva, Diniz Marina Aleixo, Leonardo Kizie Conrado, Kusumota Luciana, Haas Vanderlei José, Rodrigues Rosalina Aparecida Partezani. Síndrome de fragilidade relacionada à incapacidade funcional no idoso. Acta paul. enferm. [Internet]. 2012 [cited $2020 \mathrm{Apr} 10$ ] ; 25 ( 4 ): 589$594 . \quad$ Available from: http: //www.scielo.br/scielo.php?script=sci_arttext\&pid=S010321002012000400017\&lng=en. $\quad$ Epub July 31, 2012. https://doi.org/10.1590/S0103-21002012005000016.

4. Certo, Ana; Sanhez; Kleiver; Galvão, Ana Maria; Fernades, Helder (2016). A síndrome da fragilidade nos idosos: revisão da literatura. In Actas de Gerontologia: Congresso Português de Avaliação e Intervenção em Gerontologia Social. ISSN 2182-9314. 2:1. p.1-11[Internet]. Biblioteca digital IBP [cited 2020 Apr 16]. Available https://bibliotecadigital.ipb.pt/handle/10198/12983

5. Fabrício-Wehbe Suzele Cristina Coelho, Cruz Idiane Rosset, Haas Vanderlei José, Diniz Marina Aleixo, Dantas Rosana Aparecida Spadoti, Rodrigues Rosalina Aparecida Partezani. Reprodutibilidade da versão brasileira adaptada da Edmonton Frail Scale para idosos residentes na comunidade . Rev. LatinoAm. Enfermagem [Internet]. 2013 Dec [cited 2020 Apr 06] ; 21( 6 ) : 1330-1336. Available from: http://www.scielo.br/scielo.php?script=sci_arttext\&pid=S0104- 11692013000601330\&lng=en. http://dx.doi.org/10.1590/01041169.2933.2371.

6. Fhon Jack Roberto Silva, Rodrigues Rosalina Aparecida Partezani, Santos Jair Lício Ferreira, Diniz Marina Aleixo, Santos Emanuella Barros dos, Almeida Vanessa Costa et al . Fatores associados à fragilidade em idosos: estudo longitudinal. Rev. Saúde Pública [Internet]. 2018 [cited 2020 Apr 06] ; 52: 74. Available from:

http: / / www.scielo.br/scielo.php?script=sci_arttext\&pid=S003489102018000100266\&lng=en. Epub July 26, 2018. http://dx.doi.org/10.11606/s1518-8787.2018052000497.

7. Tinetti, M.E. (1986), Performance-Oriented Assessment of Mobility Problems in Elderly Patients. Journal of the American Geriatrics Society, 34: 119-126. [Internet]. [cited 2020 Apr 06] Available from: https://doi.org/10.1111/j.15325415.1986.tb05480.x

8. Spirduso, Waneen W. Dimensões físicas do envelhecimento. $1^{\mathrm{a}}$ Edição. Barueri (SP): Manole. 2005.

9. Andrade SR, Ruoff AB, Piccoli T, Schmitt MD, Ferreira A, Xavier AC. O Estudo de Caso como Método De Pesquisa Em Enfermagem: Uma Revisão Integrativa. Texto Contexto Enferm (Internet), 2017/2015 [cited 2020 Apr 06] ;26(4):e5360016. Available from: https: //www.scielo.br/pdf/tce/v26n4/0104-0707-tce-26-04e5360016.pdf

10. Assembleia do Colégio da Especialidade de Enfermagem de Reabilitação. Padrão Documental dos Cuidados de Enfermagem da Especialidade de Enfermagem de Reabilitação. Porto: Ordem dos Enfermeiros; 2015 [cited 2020 Apr 06]). Available from: https://www.ordemenfermeiros.pt/arquivo/colegios/Document s/2015/MCEER_Assembleia/PadraoDocumental_EER.pdf

11. Huber, F. \& Wells, C. Exercícios Terapêuticos. Planeamento do Tratamento para progressão. $1^{\text {a }}$ edição. Loures. Lusociência. 2008.

12. Ferguson, Brad. ACSM's Guidelines for Exercise Testing and Prescription 9th Ed. 2014. The Journal of the Canadian Chiropractic Association vol. 58,3 (2014): 328. Available from: http://antoinedl.com/fichiers/public/ACSM-guidelines2014.pdf.

13. Faria, J. D. C., Machala, C. C., Dias, R. C., \& Dias, J. M. D. Importância do treinamento de força na reabilitação da função muscular, equilíbrio e mobilidade de idosos. Dezembro 2003. pp.133-137.; (Internet); [cited 2020 Apr 06] Available from: https://doi.org/10.11606/issn.2317-0190.v10i3a102461

14. American College of Sports Medicine., and Leonard A. Kaminsky. ACSM's Resource Manual for Guidelines for Exercise Testing and Prescription. 5th ed. Baltimore, MD: Lippincott Williams \& Wilkins, 2006.

15. Ferreira MS, Sonoda LT, Barbosa SA, Franco FGM, Carvalho JAM. Reabilitação física na síndrome de fragilidade do idoso. Acta Fisiátr. 2014;21(1):26-28

16. Marcela Ganzella, Marcia Maria Fonto Zago. A alta hospitalar na avaliação de pacientes e cuidadores: uma revisão integrativa da literatura. Acta Paul Enferm 2008;21(2):351-5. 2007.

17. Arantes Paula M. M., Alencar Mariana A., Dias Rosângela C., Dias João Marcos D., Pereira Leani S. M.. Atuação da fisioterapia na síndrome de fragilidade: revisão sistemática. Rev. bras. fisioter. [Internet]. 2009 Oct [Cited 2020 Apr 17]; 13(5): 365-375. Available from: http: / / www.scielo.br/scielo.php?script=sci_arttext\&pid=S1413$35552009000500002 \&$ Ing $=e n$. Epub Nov 13, 2009. http://dx.doi.org/10.1590/S1413-35552009005000061.

18. Macedo, Camila, Gazzola, Juliana, Najas, Myrian. Síndrome da fragilidade no idoso: importância da fisioterapia. Volume 33. Arquivos Brasileiros de Ciências da Saúde. 2008/12/30, disponível em: https://www.portalnepas.org.br/abcs/article/view/154.

19. Andrade Ankilma do Nascimento, Fernandes Maria das Graças Melo, Nóbrega Maria Miriam Lima da, Garcia Telma Ribeiro, Costa Kátia Nêyla de Freitas Macêdo. Análise do conceito fragilidade em idosos. Texto contexto - enferm. [Internet]. 2012 Dec [cited. 2020 May 1]; 21(4): 748-756. Available from: http: / / www.scielo.br/scielo.php?script=sci_arttext\&pid=S010407072012000400004\&lng=en. https://doi.org/10.1590/S010407072012000400004.

20. Paulo Henrique Meira Duarte, Laura de Sousa Gomes Veloso, Jéssica Priscila Neres Carvalho, Bruna de Oliveira Abreu, Maria Adelaide Silva Paredes Moreira. Efeitos de um programa 
fisioterapêutico direcionados a idosos frágeis institucionalizados: uma revisão integrativa. Conbracis.

21. Preto Leonel São Romão, Gomes Joana Rita Lamas, Novo André Filipe Morais Pinto, Mendes Maria Eugénia Rodrigues, GraneroMolina José. Efeitos de um Programa de Enfermagem de Reabilitação na Aptidão Funcional de Idosos Institucionalizados. Rev. Enf. Ref. [Internet]. 2016 Mar [citado 2020 Apr 06]

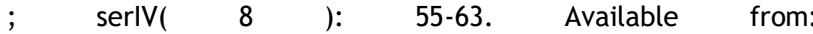
http: / / www.scielo.mec.pt/scielo.php?script=sci_arttext\&pid=S0 874-

02832016000100007\&lng=pt. http://dx.doi.org/10.12707/RIV15 019.

22. Andrade Ankilma do Nascimento, Fernandes Maria das Graças Melo, Nóbrega Maria Miriam Lima da, Garcia Telma Ribeiro, Costa Kátia Nêyla de Freitas Macêdo. Análise do conceito fragilidade em idosos. Texto contexto - enferm. [Internet]. 2012 Dec [cited $2020 \mathrm{Apr}$ 06] ; 21( 4 ): 748-756. Available from: http: / / www.scielo.br/scielo.php?script=sci_arttext\&pid=S010407072012000400004\&lng=en. https://doi.org/10.1590/S010407072012000400004. 